\title{
Tesis
}

\section{Estado-empresas transnacionales: cam- bios en el régimen de solución de contro- versias inversor-Estado y redefinición de la soberanía a la luz de la protección de los derechos humanos}

\author{
Magdalena Bas Vilizzio
}

Resumen: En medio de la crisis de legitimidad del régimen de solución de controversias inversor-Estado, la dinámica de la relación Estado-empresas transnacionales impacta en tres áreas. En primer lugar, permea en la conducta de los Estados que, a posteriori de controversias emblemáticas o congelamiento normativo, abandonaron el régimen o propusieron acuerdos "balanceados". Asimismo, la dinámica se retroalimenta con los casos concretos de controversias, especialmente aquellos que involucran temas de derechos humanos (medioambiente sano, salud pública, derecho humano al agua, etc.). Y, finalmente, se manifiesta en la transición hacia el nuevo concepto de soberanía (soberanía regulatoria), con base en la protección del espacio de política pública, cuyo centro es el derecho de regular, especialmente en materia de derechos humanos y otras áreas de interés público.

Palabras clave: Estado, Empresa transnacional, Solución de controversias inversor-Estado, Soberanía

* Cómo citar este artículo: Bas Vilizzio, M. (2020). Estado-empresas transnacionales: cambios en el régimen de solución de controversias inversor-Estado y redefinición de la soberanía a la luz de la protección de los derechos humanos. Relaciones Internacionales, 29(59), 116. https://doi.org/10.24215/23142766e116

Editor: Juan Alberto Rial, Instituto de Relaciones Internacionales Facultad de Ciencias Jurídicas y Sociales (Universidad Nacional de La Plata)
Entidad editora: Relaciones

Internacionales, es una publicación del Instituto de Relaciones Internacionales (Facultad de Ciencias Jurídicas y Sociales (Universidad Nacional de La Plata Argentina) Vol 29 - no 59/2020 


\title{
State-translational businesses: changes in dispute resolution frameworks regarding investors and the State. Redefining sovereignty in the light of the protection of human rights1
}

\section{Magdalena Bas Vilizzio2}

\begin{abstract}
In the midst of the legitimacy crisis of investor-State dispute resolution frameworks, the dynamics of the relationship between States and transnational businesses impacts on three areas. Firstly, it permeates the behavior of States that, after emblematic disputes or regulatory chills, abandoned the framework or suggested "balanced" agreements. Secondly, the dynamics are fed back with specific disputes, especially those involving human rights (healthy environment, public health, human right to water, etc.). Finally, it manifests in the transition towards the new concept of sovereignty (regulatory sovereignty) based on the protection of public policy space, whose heart is the right to regulate, especially in matters of human rights and public interest.
\end{abstract}

Key words: State, Transnational businesses, Investor-State Dispute Resolution, Sovereignty

1 Resumen de la tesis del Doctorado en Relaciones Internacionales de la Universidad Nacional de La Plata presentada por la autora y dirigida por LINCOLN BIZZOZERO. La version completa de la misma esta publicada en la pagina del IRI

2 Doctora en Relaciones Internacionales (Universidad Nacional de La Plata), Profesora Adjunta de Derecho Internacional Público y Comercio Exterior de la Universidad de la República (Uruguay). Integrante del Sistema Nacional de Investigadores (Uruguay). E-mail: magdalena.bas@fder.edu.uy 


\section{Punto de partida}

El régimen internacional de protección de inversiones, en el que se enmarca el régimen internacional de solución de controversias inversor-Estado, tuvo su momento de auge en la década de los noventa con la celebración de casi la mitad de los acuerdos actuales, gracias a su impulso por el consenso de Washington y otras instituciones vinculadas a la gobernanza del régimen, como la Conferencia de las Naciones Unidas sobre Comercio y Desarrollo (UNCTAD por su sigla en inglés). A pesar de haberse construido una red de más de 3500 acuerdos en materia de inversiones3, que establecen prórroga de jurisdicción al arbitraje internacional inversor-Estado, los mecanismos de solución de controversias inversor-Estado comenzaron a emplease de manera más dinámica a partir de la primera década del siglo XXI: de los 1061 casos conocidos, 326 corresponden a la primera década del siglo XXI y 653 a la segunda.

En esta evolución actúan como catalizadores las crisis económicas atravesadas por los dos principales Estados demandados. Este es el caso de Argentina, Estado número 1 en el ranking del Policy Investment Hub de la UNCTAD (62 demandas)4. Las medidas tomadas para paliar los efectos de crisis económica del año 2001 dan lugar a 37 de demandas de inversores extranjeros, en particular producto de las normas que imponen la conversión a pesos argentinos de los precios y tasas en contratos públicos y el abandono de las cláusulas de ajuste en monedas extranjeras o parámetros internacionales.

La situación de Argentina no es diferente a la de España, Estado número 3 en el ranking (52 demandas)5. Puntualmente, el paralelismo se puede trazar con las 47 controversias en el sector energías renovables, particularmente energía solar. Las demandas tienen como base los recortes y posterior eliminación de los beneficios a las energías renovables que forman parte del paquete de medidas tomado por el Estado para mitigar los efectos de la crisis económica 2008-2014. La problemática de España y los arbitrajes de las energías renovables generan un nuevo aumento en el número de demandas registradas, en particular en 2015, año en que se inician 83 arbitrajes, 18 correspondientes a las demandas a España.

En la actualidad, el régimen de solución de controversias inversor-Estado atraviesa una crisis de legitimidad. Entre las críticas recibidas se destaca que las demandas o amenazas de demandas de inversores extranjeros inciden negativamente sobre el espacio de política pública del Estado, especialmente sobre su corazón: el derecho de regular. Este efecto se da, en primer lugar, en términos de congelamiento o parálisis normativa dentro del propio Estado u otros que, para evitar verse involucrados en disputas, se abstienen de regular, fenómeno que es advertido en el "Cuarto reporte del Experto Independiente sobre la promoción de un orden internacional democrático y equitativo" (ONU 2015). En segundo lugar, los tribunales arbitrales se han convertido en órganos de contralor externo de la actividad o

\footnotetext{
3 Entre tratados bilaterales de inversiones (TBI), capítulos de inversiones en tratados de libre comercio, capítulos de inversiones en tratados plurilaterales.
}

4 Datos al 31 de julio de 2020. Fecha de consulta: 25 de noviembre de 2020.

5 Datos al 31 de julio de 2020. Fecha de consulta: 25 de noviembre de 2020. 
inactividad estatal (Hernández González, 2017; Postiga: 2013, p. 182; Van Hurten y Loughlin, 2016, p. 149), incluso políticas vinculadas a la protección de los derechos humanos (salud pública, derechos de las comunidades indígenas, acceso al agua y saneamiento, entre otros) y el medioambiente.

A la luz de lo anterior, este trabajo busca analizar la dinámica de la relación entre los principales actores vinculados a la inversión extranjera -Estados y empresas transnacionales-, en el marco del régimen de solución de controversias inversor-Estado. Desprendiéndose de dicho objetivo general, se plantearon tres objetivos específicos, a saber: 1) examinar las alternativas que se plantearon frente al régimen de solución de controversias inversorEstado (período 2007-presente), su fundamento y el rol de los actores involucrados; 2) determinar cuál es el impacto de la regulación estatal sobre temas vinculados a derechos humanos en casos concretos de controversias inversor-Estado que involucren a Estados de Sudamérica y la UE; y 3) valorar si el concepto de soberanía ha evolucionado hacia una nueva definición, que parte de supuestos diferentes a los que propone Krasner, explicativa de los cambios en el régimen de solución de controversias inversor-Estado, y en caso afirmativo identificar sus características y su fundamento.

En cuanto a la estrategia metodológica, conforme a sus objetivos esta investigación es explicativa y busca establecer por qué ocurre un fenómeno internacional en concreto. En relación con la dimensión temporal, se trata de un estudio longitudinal, ya que el fenómeno es observado a lo largo de un período de tiempo extenso (2007-presente) a los efectos de comprender su propia dinámica. La elección del marco temporal responde a que el año 2007 marca uno de los hitos en el régimen: Bolivia se convirtió en el primer Estado del mundo en retirarse del Centro Internacional de Arreglo de Diferencias Relativas a Inversiones (CIADI) al denunciar el Convenio de Washington. Asimismo, el 2007 fue la antesala de la crisis global de 2008, instancia que abre una nueva etapa en la que se cuestiona el modelo de gobernanza neoliberal que, estructurado desde la década del noventa, dio cabida a la fase de construcción y auge del régimen de protección de inversiones ${ }^{6}$.

La unidad de análisis es la relación Estado-empresa transnacional, en tanto actores de las relaciones internacionales y en términos del Derecho Internacional Público: la relación existente entre el Estado como sujeto originario, pleno e indiscutido y la empresa transnacional como un sujeto nuevo, limitado y discutido. Si bien se trata de una relación de poder,

\footnotetext{
6 Sornarajah (2015) plantea que existen cuatro fases en la evolución del régimen, en cada una identifica fuerzas en pugna que forman, universalizan, construyen o destruyen el régimen internacional de protección de inversiones. De esta forma, se identifican: 1) la fase formativa caracterizada por la puja entre la protección diplomática sin restricciones (propulsada especialmente desde Estados Unidos) y la doctrina Calvo (América Latina); 2) la fase de universalización de los conflictos cuyo centro es la colisión entre la fórmula Hull relativa a la expropiación y debida compensación (Estados Unidos y aliados) y la universalización de la doctrina Calvo junto a la defensa de la soberanía permanente sobre los recursos naturales; 3 ) la fase neoliberal que tiene como foco al consenso de Washington y la construcción del régimen internacional de protección de inversiones enmarcado en un red de más de $3500 \mathrm{TBI}$; y 4) la fase actual es la que presenta la lucha entre la continuación de la fase neoliberal y la resistencia al neoliberalismo a fin que el régimen no proteja exclusivamente a la inversión extranjera.
} 
en este trabajo el foco está puesto en la dinámica de la relación en el marco del régimen de solución de controversias inversor-Estado y no en el análisis del poder per se.

En vista de lo anterior, las unidades de observación son tres: 1) la red de tratados que sostiene el régimen de solución de controversias inversor-Estado, al prever el arbitraje ad hoc como mecanismo para resolver las disputas que puedan surgir; 2) los casos concretos de controversias que son dirimidas por los tribunales arbitrales ad hoc, tanto las terminadas como las pendientes; y 3 ) las alternativas al régimen de solución de controversias inversorEstado, tanto en términos de creación de una nueva institucionalidad, tratados que plantean exclusiones sectoriales o totales del arbitraje inversor-Estado, como de disidencia (retiro del régimen al que se pertenecía). El universo de análisis es acotado por el recorte temporal (2007-presente) y geográfico (Estados de Sudamérica y la Unión Europea), sin perjuicio del aporte que pueda realizarse de elementos contrafácticos para analizar la evolución de los conceptos involucrados o brindar perspectivas desde otras regiones del globo, con el fin de enriquecer el análisis y no perder la visión sistémica que esta temática requiere.

Este artículo se estructura en cuatro partes: en primer lugar se presenta el marco teórico de la investigación (apartado 2) y a continuación se dedica un apartado a cada objetivo específico con sus respectivas conclusiones parciales (apartados 3, 4 y 5). El trabajo cierra con unas reflexiones finales y un epílogo a modo de actualización al mes de noviembre de 2020.

\section{Articulación de un marco teórico para la relación Estado-empresas trans- nacionales}

El diseño de investigación es un protocolo de todas las tareas que demanda la ejecución de la investigación, por tanto comprende un conjunto de decisiones interconectadas relativas al marco teórico, los objetivos y la metodología (Sautú et al., 2005). Habiendo hecho referencia a los dos últimos, este apartado se dedicará al primero.

El marco teórico articulado para esta investigación parte de la teoría de los regímenes internacionales a los efectos de comprender el funcionamiento del sistema internacional, en lo que concierne a las relaciones entre Estados y empresas transnacionales, puntualmente en el marco del régimen de solución de controversias inversor-Estado. Este, como parte de un régimen mayor, el de protección internacional de inversiones, puede ser definido como "un conjunto implícito o explícito de principios, normas, reglas y procedimientos de toma de decisión alrededor de los cuales las expectativas de los distintos actores convergen en un área determinada de las relaciones internacionales" (Krasner, 1983, p. 2).

Desde el punto de vista jurídico, los regímenes están conformados por normas jurídicas que recogen principios y reglas que no necesariamente son compartidos por otros regímenes. Este fenómeno, actualmente se conoce con el nombre de fragmentación del Derecho Internacional General en regímenes autocontenidos o especiales, cuyos riesgos son analizados por la Comisión de Derecho Internacional de la Asamblea General (ONU, 2006). La extensión de los temas susceptibles de regulación internacional retroalimenta la existencia 
de los regímenes y una potencial tensión cuando las normas en vigor que componen cada uno de los conjuntos brindan soluciones opuestas.

Siguiendo el análisis de los modelos en pugna en el sistema internacional propuesto por Consani (2008.2016)7, en el régimen de solución de controversias inversor-Estado, el modelo institucional de las relaciones internacionales se presenta de manera preponderante, dado su alto grado de institucionalización y subordinación del poder al Derecho. Sin embargo, el modelo relacional continúa ejerciendo tensiones, que no son más que manifestaciones de la soberanía de los Estados8. De hecho, la elección de ingresar, permanecer o abandonar un régimen está directamente vinculada al atributo de la soberanía, concepto ha tenido a lo largo del tiempo: soberanía de interdependencia, interna, westfaliana y legal internacional (Krasner, 2001).

Ahora bien, no debe confundirse soberanía, como atributo de los Estados, con autonomía, esto es, "la máxima capacidad de decisión propia que se puede tener, tomando en cuenta los condicionamientos del mundo real" (Puig, 1980: 148). Es el efectivo goce de la autonomía, y no necesariamente el atributo de la soberanía, lo que le permite al Estado articular y lograr objetivos de manera independiente de las demandas o intereses de entidades internas o externas (Russell y Tokatlian, 2001). Por consiguiente y respecto a los mecanismos de solución de controversias inversor-Estado, cobra especial interés el planteo de Rapoport y Miguez (2014) respecto a la pérdida de poder de decisión de los Estados durante el período de auge del consenso de Washington y la necesidad de recuperar el control sobre los movimientos de capital y las inversiones, entre otros puntos, para fortalecer la relación de los Estados de la región con el resto del mundo.

La capacidad de acción de los Estados también puede verse menguada frente a las presiones de empresas transnacionales, por intermedio de demandas o amenazas de demandas y la consecuente parálisis o congelamiento normativo. Esto "podría disuadir en el futuro a los Estados, de tomar medidas para respetar, proteger y cumplir sus obligaciones en materia de derechos humanos y, por lo tanto, tiene un efecto negativo en el orden internacional democrático y equitativo" (ONU, 2015, p. 12), al tiempo de posicionar a las empresas transnacionales como creadoras de normas jurídicas (Arato, 2015: 283). Es en este punto que puede visualizarse un cambio en el equilibro de poder entre Estados y empresas transnacionales (Strange, 2001). El fenómeno del posible condicionamiento del espacio de política pública (base de la soberanía), por parte del régimen internacional de solución de controversias inversor-Estado (UNCTAD, 2003, p. 145), se manifiesta de manera diferente a la intervención en sentido militar (erosión de la soberanía westfaliana). No obstante, se percibe una pérdida progresiva de la autoridad real del Estado, al minimizar sus competencias

\footnotetext{
7 Consani plantea un abordaje dialéctico abierto entre el poder y el Derecho en el cual se enmarcan ambos modelos, dado que los modelos son ideales y no se presentan de manera pura en las relaciones internacionales.

8 Un estudio específico del régimen de solución de controversias inversor-Estado desde la perspectiva de los dos modelos de las relaciones internacionales puede verse en Bas Vilizzio (2018).
} 
en términos de Strange (2001, p. 73-74) y autolimitarse buscando inversiones de empresas transnacionales (Bermejo García, 2015, p. 95)9.

En suma, en la ilustración 1 puede verse una aproximación en conjunto de los diferentes aportes que conforman la base teórica de este trabajo.

\section{Ilustración 1: Marco teórico, una aproximación en conjunto}

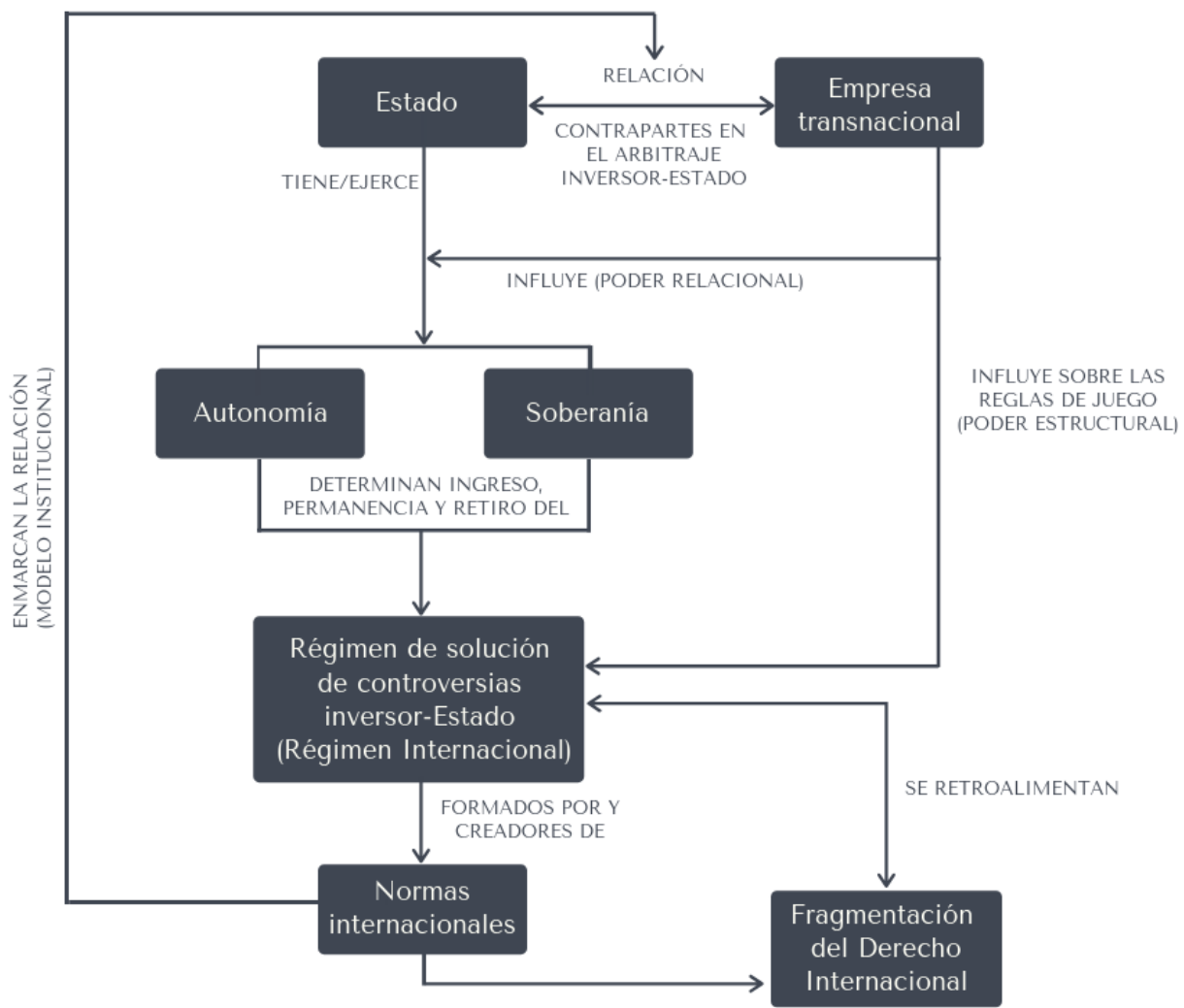

Fuente: Elaboración propia.

9 Si bien excede los objetivos de este trabajo, es pertinente señalar que se sigue la posición minoritaria de la doctrina minoritaria que afirma que las empresas transnacionales pueden ser consideradas sujetos de Derecho Internacional Público, aunque de carácter menor o limitado. Su subjetividad es oponible al Estado que las haya reconocido (no es erga omnes), si bien en el marco de un TBI u otro acuerdo de protección de inversiones, un Estado reconoce virtualmente a todas las empresas nacionales del otro Estado parte, cuyas inversiones en su territorio son protegidas. Asimismo, la subjetividad de las empresas se focaliza en las disposiciones de los instrumentos jurídicos en los que se enmarquen sus derechos y obligaciones. 


\section{Alternativas al régimen internacional de solución de controversias inver- sor-Estado}

\section{i. Abandono del régimen de solución de controversias inversor-Estado}

Para comprender la dinámica de la relación Estado-empresas transnacionales en el marco de la solución de controversias inversor-Estado, en primer lugar, se analizan cuáles son las alternativas planteadas por los Estados frente al régimen de solución de controversias inversor-Estado (período 2007- presente), su fundamento y el rol de los actores involucrados. Las alternativas se dividieron en dos grupos: aquellas que implican el abandono del régimen y aquellas que proponen nuevos modelos de acuerdos en búsqueda de un balance o exclusión del régimen.

Hasta el año 2007, inicio del marco temporal de esta investigación, no existían antecedentes de abandono del régimen. El pionero es Bolivia en 2007 mediante la denuncia del Convenio de Washington constitutivo del CIADI, seguido en 2009 por Ecuador y en 2012 por Venezuela. Asimismo, Bolivia consolidó su proceso con la terminación de los TBI en vigor (salvo cuatro), Venezuela únicamente denunció el TBI con Países Bajos en el marco del cual se registró el mayor número de casos en su contra. La situación de Ecuador muestra una diferencia sustancial al desandar el camino recorrido. A partir de 2017, una vez presentado el Informe de la Comisión para la Auditoría Integral Ciudadana de los Tratados de Protección Recíproca de Inversiones y del Sistema de Arbitraje Internacional en Materia de Inversiones (CAITISA), Ecuador denunció los TBI que aún estaban en vigor. No obstante, el año siguiente el Gobierno de Lenin Moreno incluyó el arbitraje inversor-Estado dentro de la reforma del Código Orgánico de la Producción.

Por otro lado, Sudáfrica constituye el único disidente africano, al haber terminado diez de sus TBI luego del impacto del caso Piero Foresti (caso CIADI número ARB(AF)/07/1). Los inversores cuestionaron las medidas tomadas para paliar los efectos del apartheid y posibilitar el acceso a la población históricamente desfavorecida a la explotación minera. A los motivos coyunturales se le suman otros de carácter político-ideológico (salida del apartheid y sostenimiento de un modelo democrático de inclusión) y jurídico (aplicación a sectores concretos del derecho a la igualdad consagrado en la Constitución de 1994). En definitiva, el caso Piero Foresti es una proyección de los costos de la lucha de Sudáfrica por la igualdad.

Los disidentes sudamericanos también son movidos por la coyuntura, en particular la experiencia como demandados en arbitrajes de inversiones. Ejemplo de esta problemática son los casos Chevron III contra Ecuador (Corte Permanente de Arbitraje caso número 200702) o Aguas del Tunari contra Bolivia (CIADI caso número ARB/02/3). Ambas tienen connotaciones económicas pero también sociales y medioambientales e involucran el efectivo goce de derechos humanos, como es el derecho humano al agua.

Los disidentes del régimen de solución de controversias inversor-Estado, ya sea por abandono total o parcial, también fundan su conducta en motivos políticos. Bolivia, Ecuador y Venezuela dieron los principales pasos para la salida del sistema durante gobiernos de corte progresista: primera presidencia de Evo Morales (2006-2010), segunda presidencia de 
Rafael Correa (2009-2013) y tercera presidencia de Hugo Chávez (2007-2013). Estos priorizaron una agenda de derechos sociales, protección del medioambiente y de los recursos estratégicos (hidrocarburos y gas, particularmente). Las reformas constitucionales de Bolivia (2009) y Ecuador (2008) aportaron dos elementos que determinan cambios en su política exterior y refuerzan las posiciones estatales desde una perspectiva jurídica: la consagración de los derechos de la naturaleza (siendo las industrias extractivas el centro de la mayoría de las demandas), y la prohibición de prórroga de jurisdicción al arbitraje internacional, salvo a tribunales de procesos de integración latinoamericanos y caribeños.

Aplicando la teoría de los regímenes internacionales y la tipología creada en 2017 (miembros, externos y disidentes) (Bas Vilizzio, 2017), los cuatro Estados bajo análisis son disidentes. Esto significa que formaron parte del régimen: contaban con TBI en vigor y tres de ellos adhirieron al Convenio de Washington dejando de lado los preceptos de la doctrina Calvo. Sin embargo, en años posteriores decidieron abandonar el régimen mediante la terminación de todos o algunos de dichos instrumentos.

Inicialmente, la tipología se completó con los miembros -los Estados que son parte del régimen y no presentaron cambios en su posición (figura residual) - y los externos Estados que históricamente se ubicaron fuera del régimen. En esta investigación se reformuló la tipología y se ubicó a los Estados en un tablero según: 1) se encuentren dentro o fuera del régimen bajo análisis, y 2) hayan cambiado o mantenido su posición. De esta forma, a los miembros, disidentes y externos se agregó el grupo de los confrontadores. Estos son Estados que, sin abandonar el régimen, presentan cambios en su posición original, especialmente mediante la propuesta de creación de un régimen alternativo o paralelo o buscan un escaso empleo del actual. India y los 23 miembros de la Unión Europea firmantes del acuerdo de terminación de los TBI entre ellos (5 de mayo de 2020) se ubican en la categoría de confrontadores (véanse apartados 3.2 y 5.2 respectivamente).

\section{ii. Nuevos acuerdos: entre un nuevo equilibrio en el régimen y la exclusión del arbitraje inversor-Estado}

Además de los Estados que optaron por el abandono del régimen de solución de controversias inversor-Estado, las alternativas planteadas incluyen la celebración de tratados que buscaban un balance o exclusión del régimen. Tres ejemplos fueron identificados: los acuerdos de cooperación y facilitación de inversiones (ACFI) de Brasil, el Acuerdo Integral y Progresivo de Asociación Transpacífico (CPTPP por su sigla en inglés) y el modelo de TBI de la India de 2016.

El caso de Brasil es emblemático para Sudamérica, más allá del nuevo modelo de tratado de inversiones ACFI. Brasil es Estado del continente que históricamente ha mantenido el carácter de externo al régimen, y el nuevo modelo de tratado sostiene su posición, ya que excluye la solución de disputas iniciadas por inversores extranjeros. Los motivos constitucionales prevalecen sobre los políticos. Desde el punto de vista jurídico, en 2002 el Congreso consideró que los TBI eran contrarios a la Constitución al establecer una jurisdicción diferente a la doméstica y privilegiar más al inversor extranjero, al darle derecho a demandar al Estado ante un tribunal arbitral internacional, mientras que el inversor nacional no puede hacerlo. La misma lógica impera en los ACFI, así como el fin de defender la autonomía estatal 
con independencia de los aspectos político-ideológicos. En este sentido, la negociación de ACFI comenzó en 2015 y continúa hasta la actualidad (gobiernos de Dilma Rousseff, Michel Temer y Jair Bolsonaro). El modelo ACFI fue replicado en el Mercosur (2017) y Brasil propuso su discusión a nivel de la OMC (2018).

En segundo lugar, se identificó la situación del Acuerdo Integral y Progresivo de Asociación Transpacífico (CPTPP por su sigla en inglés), el acuerdo sigue en líneas generales los modelos más actuales de TBI en lo que respecta a los mecanismos de arbitraje inversorEstado. No obstante, tiene la particularidad de excluir las medidas de control del tabaco. La experiencia de Australia (caso Philip Morris Asia10) y el enfriamiento regulatorio en Nueva Zelanda impulsaron esta decisión. La excepción de las medidas de control del tabaco es una innovación que ya ha comenzado a replicarse en otros acuerdos, a saber: enmienda del TLC Singapur-Australia y TBI Singapur - Kazajistán. No es posible afirmar que se trata de una tendencia, pero ya no es un caso aislado.

El último ejemplo lo presenta el nuevo modelo de TBI de India (2016), que cuenta con un complejo procedimiento para agotar la jurisdicción doméstica, paso obligatorio y previo a dar inicio al arbitraje internacional. Si bien el mecanismo no impide ni prohíbe los arbitrajes, su complejidad desmotivará su inicio y operará como mecanismo de protección estatal frente a demandas infundadas. La causa más profunda que lleva a India a terminar con sus TBI y elaborar un nuevo modelo de acuerdo también es coyuntural, principalmente los efectos del caso White Industries (arbitraje no institucionalizado).

En suma, en relación con el primer objetivo de investigación, se concluye que, si bien los motivos que movieron a los Estados examinados son coyunturales, políticos y jurídicos, el centro se encuentra en la protección de su soberanía regulatoria (véase apartado 5) mediante el ejercicio de la soberanía legal internacional (que le permite celebrar o terminar tratados). De esta manera, se concreta el ejercicio pleno de la autonomía, esto es, determinar cuáles son las medidas a tomar en materia económica, social, política, cultural, etc. sin injerencia de terceros Estados ni actores no estatales. Este último punto es significativo, ya que la autonomía puede ser vulnerada por empresas transnacionales en caso de parálisis o congelamiento normativo.

Asimismo, en las dos categorías de alternativas se visualiza una tendencia al retorno del Estado a parcelas de poder de las que previamente el Estado se había retirado: prórroga de jurisdicción hacia el arbitraje internacional para resolver disputas con inversores extranjeros y elección de las áreas que no serán susceptibles de controversias en la materia. La autonomía se ve fortalecida al priorizarse la jurisdicción doméstica (Sudáfrica, Brasil) y las jurisdicciones regionales (Bolivia, Ecuador, Venezuela) o al complejizar el pasaje a la jurisdicción internacional (India).

\section{Controversias inversor-Estado que involucran a los derechos humanos}

10 Caso Corte Permanente de Arbitraje número 2012 - 2. 


\section{i. Controversias que involucran el derecho a un medioambiente sano y equilibrado}

Dentro de los aspectos coyunturales analizados en el apartado anterior, se destacan las disputas vinculadas a temas de derechos humanos, en cuyo marco impactan y colisionan normas de dos regímenes jurídicos autocontenidos o especiales: el de protección internacional de inversiones y el de protección internacional de los derechos humanos, producto de la fragmentación del Derecho Internacional. En este sentido se seleccionaron seis controversias principales, las tres primeras vinculadas al derecho humano a vivir en un medioambiente sano: 1) Pac Rim contra El Salvador (caso CIADI número ARB/09/12), con relación a la no renovación de un permiso de explotación minera; 2) Eco Oro contra Colombia (caso CIADI número ARB/16/41), relativo a la no autorización de una licencia de explotación minera en base a la Ley de protección de páramos; y 3) Masdar contra España (caso CIADI número $A R B / 14 / 1$ ), vinculado a la transición energética, en concreto a medidas contrarias a los subsidios al sector energías renovables (recortes y posterior suspensión) con el objetivo de mitigar los efectos de la crisis económica española (2008-2014).

En el segundo grupo de controversias se incluyen las disputas: 1) Philip Morris contra Uruguay (caso CIADI ARB/10/7), relativo a las medidas de control del tabaco instrumentadas en protección del derecho humano a la salud pública y en seguimiento a normas de la Organización Internacional de la Salud; 2) Bear Creek contra Perú (caso CIADI número $A R B / 14 / 21$ ), en el que el tribunal se expide sobre la vinculación entre los conflictos sociales y la no obtención de una licencia social por parte de la empresa conforme a las normas de la Organización Internacional del Trabajo; y 3) Urbaser contra Argentina (caso CIADI número $A R B / 07 / 26)$, disputa en la que por primera vez un tribunal arbitral incorpora la perspectiva de derechos humanos (derecho humano al agua) en base a la reconvención de Argentina.

Con relación al primer grupo de disputas, es esencial tener presente que el aumento de demandas en el arbitraje de inversiones entre la década de los noventa y primera década del siglo XXI coincidió con una creciente preocupación internacional por la protección del medioambiente (Sornarajah, 2015: 333). En dichas disputas entra en juego, además de las normas del régimen de protección de los derechos humanos, un principio general del Derecho Internacional como es la soberanía permanente sobre los recursos naturales. Se trata de una norma de jus cogens, esto es, una norma imperativa de Derecho Internacional General, por oposición a una norma dispositiva, como las que habitualmente se incluyen en los TBI. En consecuencia, cualquier norma que nazca en contra de una de jus cogens es absolutamente nula en aplicación del artículo 53 de la Convención de Viena de Derecho de los tratados entre Estados11.

11 En relación con los derechos humanos, Echaide (2016) afirma que estos son normas de naturaleza erga omnes (carácter objetivo, obligaciones del Estado frente a la comunidad internacional en su conjunto) a diferencia de las disposiciones derivadas de los TBI que son de carácter subjetivo al regular una relación bilateral en particular. La relación de las normas erga omnes frente a las demás normas se encuentra en términos de prioridad en su cumplimiento no refiere a la validez. Diferente sería el caso si se consideran los derechos humanos como normas de jus cogens, debate que excede los objetivos de este trabajo. 
Por otra parte, los riesgos del régimen internacional de protección de inversiones, y en particular el régimen de solución de controversias inversor-Estado, quedan de manifiesto con la erosión del espacio de política púbica, especialmente en las situaciones de congelamiento normativo constatadas. Estas se presentan tanto en el caso Pac Rim contra El Salvador (sanción por el Parlamento de la ley que prohíbe la mega minería metálica una vez conocido el laudo favorable), como en el caso Philip Morris contra Uruguay (comienzo del proceso hacia la implementación del empaque neutro de cigarrillos una vez conocido el contenido del laudo). El caso Philip Morris Asia contra Australia también es una controversia destacable en este aspecto, pero el efecto del congelamiento normativo tiene lugar en su vecino, Nueva Zelanda, quien detiene el debate parlamentario relativo al empaque neutro de cigarrillos hasta tanto no fuera emitido el laudo.

El examen del caso Masdar contra España aporta otros elementos a la investigación. En este, el inversor extranjero invoca el Tratado de la Carta de la Energía frente a la suspensión, y posterior eliminación, de los subsidios a las energías renovables. Se trata de medidas de retroceso en materia del derecho a vivir en un medioambiente sano. No se prioriza la transición hacia energías más limpias, sino medidas para alcanzar la salida de la crisis económica de España. Ahora bien, no dejan de ser medidas tomadas en ejercicio válido del derecho de regular.

\section{ii. Controversias que involucran los derechos a la salud, al agua y de los pue- blos indígenas}

En relación con el siguiente grupo de controversias analizadas, esto es, casos Philip Morris contra Uruguay, Urbaser contra Argentina y Bear Creek contra Perú, se reafirma la conclusión de que las medidas cuestionadas por los inversores extranjeros se dieron en situaciones de ejercicio válido del derecho de regular. Suponer que el ordenamiento jurídico en el cual confiaron e invirtieron iba a mantenerse incambiado no se ajusta a los hechos (sistema internacional dinámico, impactos de las políticas exteriores de otros Estados, cambios sociales y económicos) ni al Derecho (no cercenamiento de los poderes regulatorios). La puja entre estabilidad y flexibilidad debe laudarse sin perder de vista el atributo de la soberanía.

Adicionalmente, los tres casos traen al debate el cuestionamiento sobre cómo hacer valer los derechos humanos por parte de los Estados. Las demandas reconvencionales no siempre están previstas en los acuerdos internacionales en los que se basa la controversia; por tanto, otros caminos pueden darse en la presentación de escritos amici curiae, pero su peso durante el arbitraje no es asimilable al de una demanda o contrademanda. El proyecto revisado del Instrumento jurídicamente vinculante para regular, en el marco del Derecho Internacional de los derechos humanos (2019), las actividades de las empresas transnacionales y otras empresas, establece la competencia de los tribunales domésticos. Sin embargo, aún si se lograra construir una jurisprudencia sólida y con principios comunes en un grupo relevante de Estados, un tribunal doméstico no tendrá el mismo impacto en la conducta de las empresas transnacionales que la creación de una institución internacional que entienda 
en la materia y se encargue de cruzar ambos regímenes internacionales (protección de los derechos humanos y protección de las inversiones) 12.

Para el caso Bear Creek puede señalarse otro aspecto fundamental: el régimen no prevé la participación de las comunidades locales, esto es, aquellos grupos humanos que reciben el impacto directo del desarrollo de las actividades vinculadas a la inversión. Aun si el Derecho Internacional de las Inversiones es relacional, esto es, define y regula las relaciones entre los actores involucrados y afectados por la inversión extranjera, las comunidades locales son un "actor ausente" (Perrone, 2019, p. 16). Su participación se ha limitado a la presentación de amici curiae durante un proceso arbitral, ya que no tienen locus standi ante los tribunales de inversiones. Sin embargo, en disputas vinculadas a minería, petróleo y aguas las comunidades locales han jugado un rol central en el origen o desarrollo de la controversia. Ejemplo de lo anterior son las controversias bajo análisis, así como Chevron contra Ecuador y Aguas del Tunari contra Bolivia, respectivamente.

Siguiendo la lógica del proyecto revisado del Instrumento jurídicamente vinculante para regular, en el marco del Derecho Internacional de los derechos humanos, las actividades de las empresas transnacionales y otras empresas (2019), son los Estados los titulares de la obligación de proteger los derechos humanos. Dado que deben contemplarse los límites al espacio de política pública, por tanto es posible afirmar junto a Perrone que

Es verdad que el Derecho Internacional de las Inversiones no impide que los Estados regulen para proteger los intereses de las comunidades locales ni impide que los tribunales locales juzguen que los inversores extranjeros son responsables de los daños. Pero hay limitaciones a esto, porque los Estados necesitan crear un buen clima de inversión, atraer inversores extranjeros $y$, en última instancia, enfrentar restricciones financieras para compensar a los inversores extranjeros, incluso cuando son conscientes de la mala conducta del inversor extranjero.13 (2019, p. 21).

En vista de lo anterior, con respecto al objetivo específico número dos, como primera conclusión se retoma el abordaje de Strange respecto al poder ganado por las empresas transnacionales que les ha permitido iniciar arbitrajes a los Estados receptores de la inversión, aun en caso de medidas en protección de los derechos humanos y otras que hacen al derecho de regular. De esta manera, se reafirma la idea de que los tribunales arbitrales ad hoc se comportan como órganos de contralor externo de los actos u omisiones estatales, como plantean autores como Hernández González (2017), Postiga (2013, p. 182) y Van Hurten y Loughlin (2016, p. 149).

12 Si bien un nuevo balance del régimen internacional de protección de inversiones necesariamente debe ir de la mano de un instrumento de tales características, este debería forjarse dentro del propio régimen para evitar una visión fragmentada y autonómica entre ambos.

13 Texto original en inglés: "It is true that international investment law does not prevent states from regulating to protect local community interests or hinder local courts from finding foreign investors responsible for damages. But there are limitations to this because states need to create a good investment climate, attract foreign investors and, ultimately, face financial constraints to compensate foreign investors, as where they were aware of the foreign investor's misconduct." 
Las disputas que involucran derechos fundamentales se destacan como leading cases, dadas sus implicancias sistémicas y domésticas en términos de erosión del derecho de regular. Sin embargo, el cuestionamiento corporativo no se focaliza exclusivamente en esta materia y, cuando lo hace, no distingue entre medidas de avance en la protección (ejemplo: caso Philip Morris contra Uruguay) o de retroceso (ejemplo: caso Masdar contra España). Esto lleva a la tercera conclusión, que refiere al medio más eficiente para hacer valer la protección de los derechos humanos en mecanismos que no los tienen en cuenta. Por consiguiente, si el acuerdo internacional lo autoriza, son las demandas reconvencionales el camino más directo frente a otras posibilidades, como ser los escritos amici curiae. Esto es sin perjuicio de la relevancia de estos últimos como medio para escuchar las "voces ausentes" de los grupos directamente afectados por las inversiones extranjeras.

\section{Soberanía regulatoria: ¿transición hacia un nuevo concepto de soberanía?}

\section{i. El espacio de política pública como base de la soberanía}

Partiendo de los elementos constatados en el apartado anterior, en particular la erosión del espacio de política pública por los mecanismos de solución de controversias inversor-Estado, en este apartado se busca valorar si el concepto de soberanía ha evolucionado hacia una nueva definición, que se funda en supuestos diferentes a los que propone Krasner en "Soberanía. Hipocresía organizada" (2001), identificar sus características y su fundamento. Ninguna de las cuatro proyecciones del abordaje de Krasner, soberanía de interdependencia, soberanía westfaliana, soberanía interna y soberanía legal internacional, tiene como centro el espacio de política pública.

En su informe del año 2003 (etapa actual del régimen internacional de protección de inversiones), la UNCTAD reconoce tardíamente que los mecanismos de solución de disputas entre inversores y Estados erosionan el espacio de política pública:

Su base es el derecho de regular, una prerrogativa soberana que deriva del control del Estado sobre su territorio y es un elemento fundamental en el régimen jurídico internacional de la soberanía del Estado. A pesar que los países receptores de la inversión limitan su autonomía regulatoria como resultado de políticas de liberalización -y tienen su autonomía limitada por ser parte de un proceso más amplio de globalización económica- los acuerdos internacionales de inversiones generan problemas particulares en relación con este punto. Estos acuerdos internacionales, como otros textos legales, son especificaciones de obligaciones legales que limitan la autonomía soberana de las partes.14 (p. 145)

14 Texto original en inglés: "Its foundation is the right to regulate, a sovereign prerogative that arises out of a State's control over its own territory and that is a fundamental element in the international legal regime of State sovereignty. Although host countries already limit their regulatory autonomy as a result of liberalization policies - and have their autonomy limited as part of the wider process of economic globalization - IIAs create 
Los dos conceptos en tensión son la estabilidad (en cuanto a la protección de la inversión extranjera) y la flexibilidad (en cuanto a la protección del espacio de política pública del Estado). Esta tensión pone en evidencia el desafío que implica para los Estados, particularmente aquellos en desarrollo, lograr un balance entre la liberalización económica y las políticas de desarrollo sostenible (Ghiotto, 2017, p. 65, 69).

Los efectos de la erosión del espacio de política pública se constatan particularmente bajo el efecto de la parálisis o congelamiento normativo, esto es, la abstención de regular o continuar un proceso normativo (discusión, elaboración, sanción, implementación o entrada en vigor) frente a una demanda o amenaza de demanda. Esta es la situación de El Salvador, cuyo parlamento no aprobó la ley que prohíbe la mega minería metálica hasta tanto no tuvo un laudo a su favor (caso Pac Rim). Incluso, el congelamiento o parálisis normativa puede darse en otros Estados que, al encontrarse en una situación similar, sean disuadidos de regular. Este último fue el caso de Nueva Zelanda frente al arbitraje que Philip Morris Asia había iniciado contra Australia por el empaque neutro de cigarrillos (caso Corte Permanente de Arbitraje número 2021-2).

Sin embargo, desde el inicio del siglo XXI se perciben movimientos de transición hacia un nuevo concepto de soberanía, cuya base es el espacio de política pública y su corazón el derecho de regular: la soberanía regulatoria. El nuevo concepto tiene notas de autoridad y legitimidad, al igual que la soberanía interna, pero no cuenta con notas de control. Asimismo, es un concepto absoluto, por oposición a la afirmación de la Corte Permanente de Justicia Internacional en el caso del vapor Wimbledon (1923). La Corte sostiene que la soberanía estatal no puede verse erosionada por la asunción de compromisos internacionales, puesto que esta actividad se realiza justamente en ejercicio de la soberanía. Puntualmente, indica que:

La Corte rechaza que la celebración de cualquier tratado por el cual un Estado se obligue a hacer o no hacer determinado acto implique un abandono de su soberanía. No hay duda de que una convención que crea obligaciones de este tipo restringe el ejercicio de los derechos soberanos del Estado, en el sentido que requiere que ellos sean ejercidos de determinada manera. Pero el derecho de ser parte de compromisos internacionales es un atributo de la soberanía estatal. ${ }^{15}$ (párrafo 35 in fine)

Sin embargo, este ejercicio tiene un límite, que es el derecho de regular. La soberanía regulatoria es la que plantea el límite al accionar estatal, para justamente no ingresar en el círculo vicioso que propone la sentencia de 1923: si el Estado al celebrar tratados no erosiona su soberanía, puede desarrollar esta práctica hasta perder todas las características y

distinctive issues in this connection. Such international agreements, like other legal texts, are specifications of legal obligations that limit the sovereign autonomy of the parties."

15 Texto original en inglés: "The Court declines to see in the conclusion of any Treaty by which a State undertakes to perform or refrain from performing a particular act an abandonment of its sovereignty. No doubt any convention creating an obligation of this kind places a restriction upon the exercise of the sovereign rights of the State, in the sense that it requires them to be exercised in a certain way. But the right of entering into international engagements is an attribute of State sovereignty." 
desaparecer como tal. Por consiguiente el Estado dejaría de ser Estado, aunque la Corte afirme que los tratados no erosionan su soberanía16.

Las limitaciones, si existieran, refieren a la autonomía, como condición y no atributo del Estado. La autonomía está representada en la elección de los mecanismos que permitan al Estado desarrollarse como mejor lo entienda y, por ende, ejercer su condición de soberano. En términos jurídicos, la autonomía se manifiesta en el principio de libre determinación de los pueblos que establece el derecho de estos a elegir libremente su condición política y, por tanto, determinar de forma autónoma las políticas de desarrollo económico, social y cultural, excluida de su ejercicio cualquier forma de injerencia externa.

Las relaciones internacionales, como disciplina, están en constante movimiento. Aún no está definida la determinación de las áreas de regulación contenidas dentro del derecho de regular como corazón del espacio de política pública y que, por tanto, no pueden ser objeto de injerencia externa. La soberanía regulatoria es un concepto en construcción, sin perjuicio de que del análisis realizado se pueda inferir que debe incluirse la protección del medioambiente, la protección de los derechos humanos o la seguridad nacional como áreas generales, y la protección de la salud pública o las medidas de control del tabaco, en particular.

\section{ii. Soberanía regulatoria, un concepto en transición}

El nuevo concepto de soberanía surge en un momento histórico significativo: crisis de la globalización y crisis del multilateralismo, que retroalimentan la crisis de legitimidad del régimen de solución de controversias inversor-Estado. La primera manifestación es el retiro de Bolivia del CIADI en 2007, año previo a la crisis global de 2008 que genera un quiebre en la globalización como modelo de gobernanza neoliberal, articulado en torno a dos ideas fuerza: el debilitamiento del Estado y la dispersión del poder hacia actores no estatales junto a nuevas dinámicas de dependencia, desigualdad, dominación y exclusión (del Arenal, 2009). La globalización no se desvanece en 2008 , pero se da inicio a un momento de transición o crisis.

Este momento se manifiesta en cuatro fenómenos identificados por Sanahuja (2018), a saber: 1) modificaciones en la distribución del poder; 2) debilitamiento del modelo económico de transnacionalización de la producción basado en el off-shoring; 3) agotamiento del modelo en términos sociales y medioambientales, especialmente en lo que respecta al extractivismo y a la segregación como plantea Sassen (2011); y 4) falencias en la gobernanza doméstica e internacional.

La crisis de las instituciones de Bretton Woods - Organización Mundial del Comercio, cuna del régimen de solución de controversias inversor-Estado, genera el ambiente ideal

16 Se es soberano o no se es, en otras palabras, se es Estado o no se es, sin posibilidad de graduación (soberano al $100 \%, 90 \%, 80 \%$, etc.) 
para el momento de mayor crítica de los mecanismos arbitrales y la presentación de propuestas alternativas. Es en este marco que comienza a gestarse el nuevo concepto de soberanía, al constatar los Estados su erosión y plantear la necesidad de cambios profundos.

¿Es la solución de controversias el catalizador de la necesidad de reforma del régimen internacional de protección de inversiones? Esta pregunta puede verse reforzada si se tiene en cuenta que en otra institución típica del multilateralismo como es la Organización Mundial del Comercio, también su sistema de solución de disputas está en crisis, en particular el Órgano de Apelaciones. Los mecanismos de solución de disputas que involucran a Estados soberanos pueden poner en jaque políticas domésticas y permear directamente en los poderes regulatorios. En otros términos, el núcleo de la respuesta está en el concepto de soberanía y la erosión del espacio de política pública por los mecanismos de solución de disputas cuando las normas sustantivas no establecen límites claros.

Ejemplos de manifestaciones de la transición hacia la soberanía regulatoria son: los casos de abandono del régimen (Bolivia, Ecuador, Venezuela y Sudáfrica, con diferente alcance cada uno), la celebración de tratados que buscan un balance (el CPTPP por la excepción de las medidas de control del tabaco, y el modelo de TBI de India de 2016 que fortalece la jurisdicción doméstica), o los tratados que excluyen los mecanismos de arbitraje inversorEstado (los ACFI de Brasil) (véase apartados 3.1 y 3.2).

Otra manifestación está representada en el texto del TBI Marruecos-Nigeria, que incorpora disposiciones con mayor grado de protección del espacio de política pública. Estos son ejemplos de cómo la soberanía regulatoria es un concepto que excede el ámbito doméstico de los Estados y busca transformar las instituciones internacionales (proceso que se construye desde el interior al exterior). Asimismo, a nivel de los principios que componen el régimen de solución de controversias inversor-Estado, un nuevo principio se deriva del principio general y busca limitar el principio general de protección de la inversión extranjera (principio de protección del derecho de regular). Este proceso aún no está consolidado.

La posición de la Unión Europea también puede ser señalada como ejemplo de transición hacia la soberanía regulatoria; no obstante, se trata de una posición de mayor complejidad y fragmentación. Por un lado, por efecto del caso Achmea, al interior se rechazan los arbitrajes intra-Unión Europea y sus laudos se consideran nulos. Asimismo, el 5 de mayo de 2020, 23 de los 27 miembros firmaron un acuerdo para terminar los TBI entre ellos17. EI texto del acuerdo establece la terminación de los TBI en vigor entre los firmantes (125 en total) y es innovador al establecer que las cláusulas de ultractividad no producirán efectos jurídicos. Al tiempo de lo anterior, para las relaciones con terceros Estados, los miembros de la Unión Europea mantienen los TBI en vigor, incluso el Tratado de la Carta de la Energía18, en el cual la Unión Europea es parte per se. Asimismo, a nivel de los acuerdos con

17 No son parte del tratado Austria e Irlanda, firmando de la declaración del 24 de octubre de 2019, ni Suecia y Finlandia que no acompañan el proceso negociador desde el inicio.

$18 \mathrm{El}$ tratado se encuentra en proceso de renegociación, proceso que incluye la propuesta del sistema de tribunales de inversión de la Unión Europea. 
terceros, el bloque está negociando la incorporación de un sistema de tribunales de inversiones, que, de concretarse, a mediano plazo actuará en paralelo con el régimen actual. Conforme a la reformulación de la tipología de Estados según su posición respecto al régimen, la Unión Europea junto a India son confrontadores.

El ciclo de negociación o renegociación de acuerdos comerciales y/o de inversiones con terceros Estados o grupos de Estados se encuentra en marcha. Ejemplo de lo anterior es la modernización del acuerdo con México y el Acuerdo Económico y Comercial Global (CETA) entre la Unión Europea y Canadá, cuyo capítulo vinculado al sistema de tribunales de inversión no está en vigor y tiene fuerte resistencia de parlamentos locales belgas. Por otra parte, en el Acuerdo Mercosur-Unión Europea se excluyeron los mecanismos tradicionales de arbitraje de inversiones 19 y, a nivel multilateral, no se han avanzado con la negociación de un sistema multilateral de tribunales de inversiones 20.

En suma, la soberanía es un concepto que tiene plena vigencia en el sistema internacional, pero sufre transformaciones a lo largo de la historia. El concepto de soberanía regulatoria aún se encuentra en construcción. Está dando pasos para moldear las instituciones internacionales (proceso desde el interior al exterior del Estado), esto es, la reconstrucción del régimen internacional de solución de controversias inversor-Estado.

\section{Reflexiones finales}

La dinámica de la relación Estado-empresas transnacionales en el marco del régimen de solución de controversias inversor-Estado puede explicarse de manera gráfica mediante la unión de tres círculos en un diagrama de Venn (véase ilustración 2). En el primero se encuentran las alternativas al régimen actual que los Estados presentan en materia de tratados, ya sea su abandono mediante la denuncia de los acuerdos en vigor, la exclusión del régimen de los acuerdos de inversiones o la elaboración de instrumentos que buscan un mayor balance en el régimen. Los Estados involucrados en las alternativas son motivados por aspectos político-ideológicos, jurídicos (especialmente el respeto a las normas constitucionales) y coyunturales (la experiencia como demandados en arbitrajes inversor-Estado).

En función de la experiencia en el régimen, se destacan aquellas controversias las que se cuestionan políticas públicas vinculadas a la mayor protección de los derechos humanos. Sin embargo, los arbitrajes tienen como objeto el cuestionamiento de medidas de extensión de la regulación en materia de avances en derechos humanos pero también de retroceso, así como cualquier otro cambio regulatorio que realice el Estado. En definitiva, el cumplimiento del Estado de su función de regular impulsa a las empresas transnacionales a iniciar arbitrajes, sea cual sea el contenido concreto de dichas nuevas medidas.

Ahora bien, es la protección del espacio de política pública, o más precisamente de su núcleo (el derecho de regular), lo que lleva a constatar la transición hacia un nuevo concepto

19 Para un análisis de los motivos véase Bas Vilizzio (2019).

20 La Comisión cuenta con mandato negociador desde el año 2018. 
de soberanía: la soberanía regulatoria. Justamente, esta se basa en la protección del espacio de política pública y se manifiesta en las diferentes alternativas que han propuesto los Estados frente al régimen actual. Esto es, su abandono total o parcial, su exclusión de los acuerdos de inversiones, la celebración de acuerdos de mayor balance (exclusiones sectoriales o reforzamiento de la jurisdicción doméstica), así como nuevos acuerdos que profundizan en la protección del espacio de política pública.

\section{Ilustración 2: Relación Estado-empresas transnacionales: una síntesis}

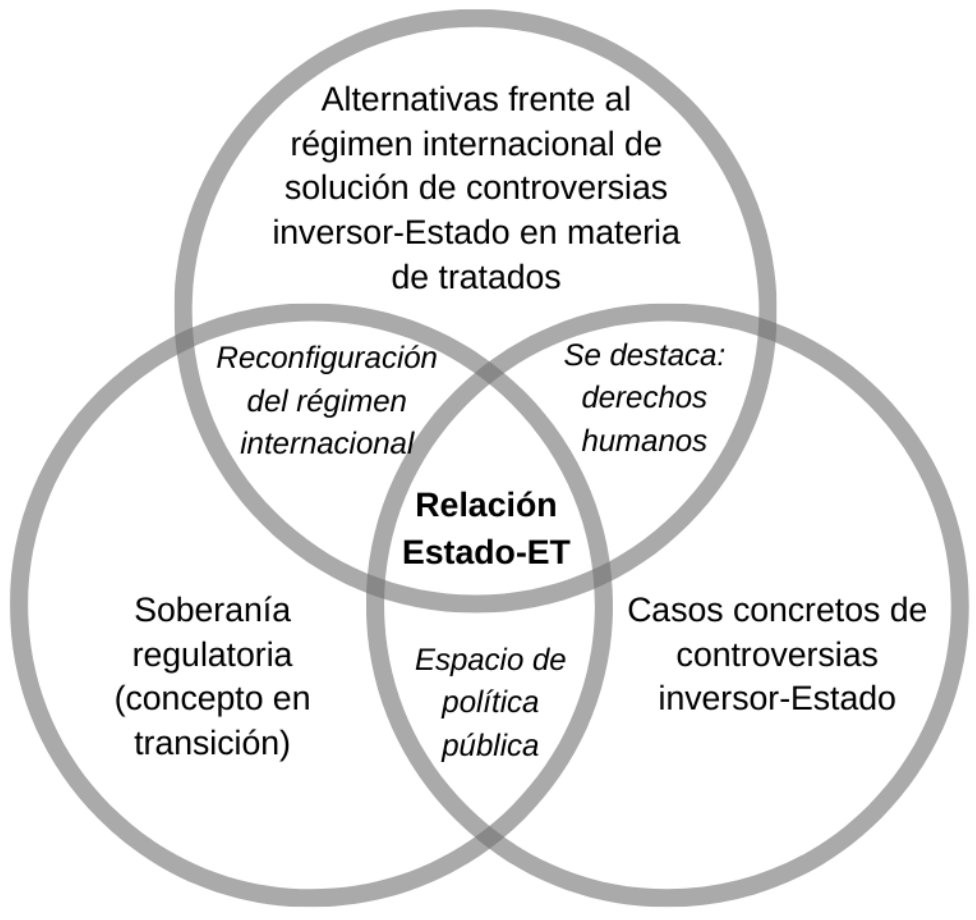

Fuente: Elaboración propia.

El concepto de soberanía regulatoria que propone este trabajo cuenta con las notas de autoridad y legitimidad, pero carece de la nota de control. La autoridad se expresa en el entendimiento de que en la soberanía regulatoria el Estado no admite invitaciones válidas para su erosión o condicionamiento, porque correría el riesgo de vulnerar su sustento: el espacio de política pública. Si existieran limitaciones, estas serían a la autonomía estatal, como condición y no atributo del Estado. En este punto el concepto se posiciona en la vereda opuesta a la clásica sentencia de la Corte Permanente de Justicia en el asunto del vapor Wimbledon. La soberanía regulatoria no se focaliza en la capacidad de elegir entre diferentes políticas públicas: esta elección está en el ámbito de la autonomía, como ejercicio del principio de libre determinación de los pueblos. 
En la actualidad, no es posible identificar con precisión un elenco de actos estatales o áreas de regulación que conformen la soberanía regulatoria, ni un criterio unívoco para determinarlo. Si bien el trazado de los límites de la soberanía regulatoria se encuentra en construcción, las normas analizadas permiten inferir que se encuentran incluidos las normas sobre seguridad estatal, medioambiente, salud, y medidas de control del tabaco, posicionando en la cúspide a la protección de los derechos humanos. Así se podría evitar el congelamiento o parálisis regulatoria en dichas áreas. Es por esto que la nota fundamental de la soberanía regulatoria es la legitimidad, tanto en términos jurídicos (defensa de un conjunto de normas) como sociológicos (apoyo de las audiencias relevantes que aceptan dichas normas como las orientadoras de la conducta a seguir).

El concepto de soberanía regulatoria, aun sin haber cristalizado su evolución, surge en un contexto de crisis de la globalización y del multilateralismo. Justamente, el régimen internacional de protección de inversiones tiene su momento de auge durante la globalización, enmarcado en una tendencia hacia la desregulación, apertura económica y privatización de la justicia propulsadas por las instituciones vinculadas a la gobernanza del sistema. En consecuencia, este punto de inflexión en el sistema actúa como catalizador de reivindicaciones soberanas y evolución hacia la protección del espacio de política pública. En este sentido, la experiencia de los Estados, como demandados en el régimen de solución de controversias inversor-Estado, ha sido uno de los elementos que marcan el camino.

En suma, sesenta años después de la celebración del primer TBI, el régimen internacional de solución de controversias inversor-Estado presenta quiebres cada vez más visibles. La relación Estado-empresas transnacionales transita entre dos ideas en pugna: la estabilidad en la protección de la inversión y la flexibilidad desde la lógica de la protección del espacio de política pública. La evolución hacia un régimen más justo y balanceado, sea cual sea el camino, necesariamente deberá tener presente la protección del espacio de política pública o correrá el riesgo de perder toda legitimidad.

\section{Epílogo}

La tesis doctoral que da origen a este artículo fue terminada en noviembre de 2019. En 2020 otra crisis irrumpe en el sistema internacional: la pandemia del nuevo coronavirus (covid-19), crisis que puede ser considerada como una crisis dentro de otra mayor -la crisis de la globalización (Sanahuja: 2020). Una pandemia -o sindemia, para considerar los factores biológicos y sociales como propone Horton (2020, p.396) -, a primera vista no tiene ninguna relación con el régimen de solución de controversias inversor-Estado. Sin embargo, las medidas adoptadas por los Estados para paliar los efectos sanitarios, económicos y sociales del virus pueden ser terreno fértil para demandas de inversores extranjeros 21 .

El informe de la UNCTAD de mayo de 2020 advierte que el posible aumento en el número de demandas tiene como motivo que gran parte de la red de acuerdos internacionales

21 Un análisis específico de los impactos de la pandemia de covid-19 en el régimen de solución de controversias inversor-Estado puede verse en Bas Vilizzio (2020). 
de inversiones fueron celebrados en momentos históricos en los que temas como el medioambiente o la salud pública tenían menos trascendencia en la agenda internacional 22. Por consiguiente, los acuerdos no suelen plantear excepciones a los arbitrajes en estas materias. En este contexto, frente a eventuales demandas podría argumentarse que las medidas adoptadas se enmarcan en la eximente de estado de necesidad como eximente de responsabilidad por incumplimiento de normas internacionales (artículo 25 de la Resolución AG/56/83). No obstante, no debe perderse de vista que la decisión siempre estará en manos de un tribunal arbitral ad hoc y que en ocasiones anteriores la jurisprudencia no ha sido consistente, incluso ante la misma situación de hecho23.

Es por este motivo que, desde la academia y la sociedad civil organizada, se propone la limitación en el acceso a los arbitrajes que tengan como base el cuestionamiento de medidas sanitarias, económicas y sociales adoptadas a fin de paliar los efectos del covid-19, así como la moratoria de los arbitrajes en curso (ACAFREMIN et al., 2020; CCSI, 2020; Gallagher y Kozul-Wright, 2020). La propuesta no solucionaría el problema de fondo; sin embargo, evitaría el rechazo de solicitudes de extensión de plazo para las actuaciones planteadas por Estados como Bolivia en el marco de los procesos en su contra24. Asimismo, fortalecería la soberanía regulatoria de los Estados que busquen acelerar los procedimientos de obtención de licencias obligatorias para medicamentos y vacunas contra el covid-19, como se ha avanzado en Alemania25, Ecuador ${ }^{26}$ y Chile $^{27}$.

\section{Referencias}
ACAFREMIN, ACTIONAID, AFRICA EUROPE FAITH \& JUSTICE NETWORK, AMIGOS DE LA TIERRA AMÉRICA LATINA Y EL CARIBE - ATALC, ARAB NGO NETWORK FOR DEVELOP- MENT, ASIA PACIFIC FORUM ON WOMEN, LAW \& DEVELOPMENT (APWLD)... ZIMBABWE INSTITUTE FOR INTERNATIONAL AFFAIRS (2020). Open Letter to Governments on ISDS and COVID-19. http://s2bnetwork.org/wp-content/up- loads/2020/06/OpenLetterOnISDSAndCOVID_June2020.pdf

ARATO, J. (2015). Corporations as Lawmakers. Harvard International Law Journal. Summer

22 El 60\% de los acuerdos fueron celebrados en la década del noventa o previamente.

23 En las disputas vinculadas a las medidas adoptadas para paliar los efectos de la crisis económica argentina del año 2001, el estado de necesidad fue reconocido en los casos LG\&E (caso CIADI número ARB/02/1) y Continental (caso CIADI número ARB/03/9), no así en los casos Enron (caso CIADI número ARB/01/3), CMS (caso CIADI número ARB/01/8) y Sempra (caso CIADI número ARB/02/16).

24 Casos Orlandini (caso CPA número 2018-39) y Glencore (caso 2016-39).

25 Enmiendas a la Ley de Prevención y control de enfermedades infecciosas en humanos y la Ley de Protección a la población en caso de epidemias de importancia nacional, 27 de marzo de 2020.

26 Resolución de la Comisión de Educación, Cultura, Ciencia y Tecnología de la Asamblea Nacional al Ministro de Salud Pública, 20 de marzo de 2020.

27 Resolución de la Cámara de Diputados número 896, modificatoria del artículo 51 de la Ley de Propiedad Industrial (Ley 19.030), 17 de marzo de 2020. 
2015. 56 (2), 229-295. Harvard University. http://www.harvardilj.org/wp-content/uploads/562Arato.pdf

BAS VILIZZIO, M. (2020). Las respuestas a la COVID-19 en el laberinto de la solución de controversias inversor-Estado. Análisis Carolina 46/2020. Fundación Carolina. https://www.fundacioncarolina.es/ac-46-2020/

(2019). Acuerdo Mercosur-Unión Europea: sombras y ausencia de la solución de controversias inversor-Estado. Documentos de Trabajo 21/2019 (2 época). Fundación Carolina. https://www.fundacioncarolina.es/wp-content/uploads/2019/11/DT_FC_21.pdf

(2018). Régimen de solución de controversias inversor-Estados: ¿resistencias del modelo relacional en un marco institucional de las relaciones internacionales? Revista Relaciones Internacionales, (54), 163-178. Instituto de Relaciones Internacionales de la Universidad Nacional de La Plata. https://revistas.unlp.edu.ar/RRII-IRI/article/view/5167/4642

(2017). América del Sur ante los tratados bilaterales de inversión: ¿hacia un retorno del Estado en la solución de controversias? Ediciones Universitarias, Comisión Sectorial de Investigación Científica - Universidad de la República.

BERMEJO GARCÍA, R. (2015). Las empresas transnacionales como actores y sujetos «potenciales» en la sociedad internacional. En: BARRANCO AVILÉS, M. CELADOR ANGÓN, O. Y VACAS FERNÁNDEZ, F. (Coord.) Perspectivas actuales de los sujetos de Derecho, 90-116. Departamento de Derecho Internacional, Universidad Carlos III.

COLUMBIA CENTER FOR SUSTAINABLE INVESTMENT (2020). Call for ISDS Moratorium During COVID-19 Crisis and Response. CCSI. http://ccsi.colum-

bia.edu/2020/05/05/isds-moratorium-during-covid-19/

CONSANI, N. (2016) Introducción. En: CONSANI, N. (Dir. y Comp.) (2016) Nuevos desarrollos del Derecho Internacional, 9-10. Instituto de Relaciones Internacionales de la Universidad Nacional de La Plata. http://www.iri.edu.ar/wp-content/uploads/2016/08/cat2-manualderecholnternacionalP\%C3\%BAblico-completo.pdf

(2008). Los modelos en la sociedad internacional contemporánea. Serie Derecho Internacional Contemporáneo (1). Instituto de Relaciones Internacionales de la Universidad Nacional de La Plata.

DEL ARENAL, C. (2009). Mundialización, creciente interdependencia y globalización en las relaciones internacionales. Cursos de Derecho Internacional de Vitoria-Gasteiz, (2), 181-268. Servicio Editorial de la universidad del País Vasco.

ECHAIDE, J. (2016). Demandas en el CIADI y el derecho humano al agua: ¿tratados de inversiones vs derechos humanos? International Law, Revista Colombiana de Derecho Internacional, (31), 81-114. Universidad Javeriana. doi.org/10.11144/Javeriana.il14-31.dcdh

GALLAGHER, K. Y KOZUL-WRIGHT, R. (2020, 26 de junio de 2020). Breaking Out of the Double 
Squeeze: The Need for Fiscal and Policy Space during the COVID-19 Crises. Global Policy Journal. https://www.globalpolicyjour-

nal.com/blog/26/06/2020/breaking-out-double-squeeze-need-fiscal-and-policy-space-during-covid-19-crises

GHIOTTO, L. (2017). ¿UNCTAD pro-desarrollo o pro-liberalización? Un estudio de los cambios en el organismo a la luz de las políticas sobre inversiones. ECHAIDE, J. (Dir.) Inversiones extranjeras y responsabilidad internacional de las empresas. Problemáticas en torno al CIADI, los TBI y los derechos humanos, 53-78. BdeF.

HERNÁNDEZ GONZÁLEZ, J. (2017). Regulación económica y arbitraje internacional de inversiones. RED: Revista Electrónica de Direito, (1). Porto: Facultad de Derecho de la Universidad de Porto. https://dialnet.unirioja.es/servlet/articulo?codigo $=6421947$ \&orden $=0$ \&info $=$ link

HORTON, R (2020). Offline: COVID-19 is not a pandemic. The Lancet, 396 (10255), 874. doi.org/10.1016/S0140-6736(20)32000-6

KRASNER, S. (2001). Soberanía, hipocresía organizada. Paidós

(1983). Structural causes and regime consequences: regimes as intervening variables. En: KRASNER, S. (Ed.) (1983). International regimes. Cornell University Press.

ONU (2015). Cuarto reporte del Experto Independiente sobre la promoción de un orden internacional democrático y equitativo. Documento $A / 70 / 285$.

http://www.un.org/en/ga/search/view_doc.asp?symbol=A/70/285\&referer=h ttp://www.ohchr.org/EN/Issues/IntOrder/Pages/IEInternationalorderIndex.as px\&Lang=S

(2006). Fragmentación del Derecho Internacional: dificultades derivadas de la diversificación y expansión del Derecho Internacional, en: ONU (2006). Anuario de la Comisión de Derecho Internacional, 58a período de sesiones de la Asamblea General, 192 - 202. http://legal.un.org/docs/index.asp?path=../ilc/reports/2006/spanish/chp12.pdf\&lang=EFSRAC\&referer=http://legal.un.org/ilc/reports/2006/

PERRONE, N. (2019). The "Invisible" Local Communities: Foreign Investor Obligations, Inclusiveness, and the International Investment Regime. AJIL Unbound, (113), 1621. doi:10.1017/aju.2018.92

POSTIGA, A. (2013). A emergência do direito administrativo global como ferramenta de regulação transnacional do investimento estrangeiro direto. Revista de Direito Internacional, 10(1), 171 - 193. UniCEUB. doi:10.5102/rdi.v10i1.2369

PUIG, J.C. (1980). Doctrinas internacionales y Autonomía latinoamericana. Universidad Simón Bolívar, Instituto de Altos Estudios de América Latina.

RAPOPORT, M. Y MIGUEZ, M. C. (2014). Desafíos y ejes para una inserción internacional autónoma de Argentina y América del Sur en el escenario mundial. En: BRICEÑO, J. Y SIMONOFF, A. (Ed.) (2014). Integración y cooperación regional en América Latina. Una relectura a partir de la teoría de la autonomía. Biblos. 
RUSSELL, R. Y TOKATLIAN, J. (2001). De la autonomía antagónica a la autonomía relacional: una mirada teórica desde el Cono Sur. PostData, (7), 71-92.

http://www.revistapostdata.com.ar/2011/12/de-la-autonomia-antagonica-ala-autonomia-relacional-una-mirada-teorica-desde-el-cono-sur-robertorussell-juan-tokatlian

SANAHUJA, J.A. (2020). COVID-19: riesgo, pandemia y crisis de gobernanza global. En: MESA, M. (Coord.) (2020). Riesgos globales y multilateralismo: el impacto de la COVID-19. Anuario CEIPAZ 2019-2020, 27-54. CEIPAZ

(2018). Crisis de globalización, crisis de hegemonía: un escenario de cambio estructural para América Latina y el. Caribe. América Latina y el Caribe frente a un Nuevo Orden Mundial: Poder, globalización y respuestas regionales. Colección Pensamiento Propio, 7-68. Icaria Editorial. http://www.cries.org/wp-content/uploads/2018/06/007-Sanahuja.pdf

SASSEN, S. (2011). A Savage Sorting of Winners and Losers, and Beyond. CALHOUN, C., Y DERLUGUIAN, G. (Eds.). (2011). Aftermath: A New Global Economic Order? NYU Press.

SAUTÚ, R., DALLE, P., BONIOLO, P., Y ELBERT, R. (2005). Construcción del marco teórico, formulación de los objetivos y elección de la metodología. CLACSO.

SORNARAJAH, M. (2015). Resistance and Change in the International Law on Foreign Investment. Cambridge University Press.

STRANGE, S. (2001). La retirada del Estado. La difusión del poder en la economía mundial. Encuentro. Icaria Editorial. Intermón Oxfam.

UNCTAD (2003). World Investment Report 2003. FDI Policies for Development: National and International Perspectives. UNCTAD. https://unctad.org/en/Docs/wir2003light_en.pdf

VAN HARTEN, G. Y LOUGHLIN, M. (2006). Investment Treaty Arbitration as a Species of Global Administrative Law. The European Journal of International Law, 17(1), 121150. 10.1093/ejil/chi159 